\title{
Hyponatremia in Cirrhosis
}

\author{
Samuel H. Sigal, MD
}

Department of Medicine, NYU Langone Medical Center, New York, New York.

Hyponatremia is common in patients with cirrhosis. Portal hypertension, diuretics, large volume paracentesis without albumin, infection, and multiple medications are connected with the development or worsening of hyponatremia. Hyponatremia in cirrhosis, particularly advanced cirrhosis, is a common development. ${ }^{1}$ In a population survey of 997 patients with cirrhosis, 486 (49.4\%) and 211 (21.6\%) had a serum sodium concentration $\left(\left[\mathrm{Na}^{+}\right]\right)<135 \mathrm{mEq} / \mathrm{L}$ and $\leq 130$
$\mathrm{mEq} / \mathrm{L}$, respectively. ${ }^{2}$ Hyponatremia and its severity correlate with the presence of severe complications of cirrhosis such as hepatorenal syndrome, encephalopathy, and spontaneous bacterial peritonitis. The presence of hyponatremia frequently complicates the management of the cirrhotic patient. Journal of Hospital Medicine 2012;7:S14-S17 (C) 2012 Society of Hospital Medicine
Cirrhosis is one of the main causes of hypervolemic hyponatremia, a dilutional form of hyponatremia that occurs when there is an increase in total body water but a relatively smaller increase in total serum sodium. Portal hypertension is the main precipitating factor in fluid retention that leads to the development of cirrhotic hyponatremia. In cirrhosis, portal hypertension is determined by 2 main factors: increased intrahepatic resistance and increased spanchnic blood flow. The increased intrahepatic resistance is due to both structural (fibrosis, conversion of low resistance fenestrated sinusoids into capillaries) and dynamic (vasoconstriction due to endothelial cell dysfunction) changes. ${ }^{1}$

The hepatic circulation normally is able to accommodate an increase in portal blood flow associated with postprandial hyperemia. The elevated intrahepatic resistance in cirrhosis results in an inability to accommodate the normal increase in portal blood flow that occurs in the postprandial hyperemia state. ${ }^{3}$ As a result, portal pressure increases during postprandial hyperemia, leading to reflex vasoconstriction, which creates a shear stress and increases splanchnic nitric oxide (NO) production. ${ }^{4} \mathrm{NO}$, one of the most important vasodilators in the splanchnic circulation, increases splanchnic blood flow and portal pressures. When this happens repeatedly, it leads to a progressive dilation of preexisting portosystemic vascular channels and the development of varices. ${ }^{5}$ At the same time, levels of vascular endothelial growth factor rise;

*Address for correspondence and reprint requests: Samuel H. Sigal, Associate Clinical Professor of Medicine, NYU Langone Medical Center, 530 First Ave, New York, NY 10016; Telephone: 212-263-3643; Fax: 212-263-3751; E-mail: Samuel.sigal@nyumc.org

Additional Supporting Information may be found in the online version of this article.

Received: September 9, 2011; Revised: December 14, 2011; Accepted: January 1, 2012

2012 Society of Hospital Medicine DOI 10.1002/jhm.1915

Published online in Wiley Online Library (Wileyonlinelibrary.com). this is a very important mediator for angiogenesis because it increases NO, further increasing splanchnic vasodilation. $^{6}$

Progressive splanchnic vasodilation and increased blood flow into the splanchnic circulation leads to central hypovolemia, arterial underfilling, and decreased blood flow in renal afferent arterioles. Vasoconstrictor norepinephrine and antinatriuretic mechanisms are subsequently activated in an attempt to normalize renal perfusion pressures. Baroreceptormediated nonosmotic release of arginine vasopressin (AVP) is triggered and renin angiotensin-aldosterone system activity is increased, which increases sodium reabsorption and activates the stellate cells, causing fibrosis, vasoconstriction, and increased portal pressures. ${ }^{6,7}$

AVP acts at vasopression-1A $\left(\mathrm{V}_{1 \mathrm{~A}}\right)$ receptors to increase arterial vasoconstriction, and at $\mathrm{V}_{2}$ receptors in renal tubule cells for solute-free water retention. ${ }^{1}$ The increased sodium and water reabsorption leads to fluid retention, increased central blood volume, venous return to the heart, and an increase in cardiac output to maintain arterial perfusion and create the hyperdynamic circulation that is characteristic of cirrhosis with advanced portal hypertension. Dilutional hyponatremia develops when free water retention is more pronounced than that of sodium retention.

\section{CLINICAL FACTORS ASSOCIATED WITH CIRRHOTIC HYPONATREMIA}

Diuretics lead to hyponatremia through several mechanisms. ${ }^{8}$ First, they induce a contraction of the central blood volume, leading to the nonosmotic release of AVP. In advanced cirrhosis, there is activation of the renin-angiotensin system in addition to the nonosmotic release of AVP, leading to sodium and free water reabsorption. Diuretics block the sodium reabsorption. However, the water-retaining effects persist, further contributing to dilutional hyponatremia. ${ }^{8}$ This cycle is made worse by low sodium intake and 
frequent thirst experienced by these patients. ${ }^{8}$ Other medications (eg, non-steroidal anti-inflammatory drugs, proton pump inhibitors, and selective serotonin reuptake inhibitors) commonly prescribed for cirrhotic patients may also contribute to the development or worsening of dilutional hyponatremia. ${ }^{8}$

Increased intrathoracic pressure in patients with tense ascites can also contribute to dilutional hyponatremia by increasing baroreceptor-mediated release of AVP. ${ }^{9}$ Large volume paracentesis without the oncotic influence of albumin, an intervention commonly required in patients with cirrhosis and recurrent ascites, may also lead to significant increases in plasma renin activity and plasma aldosterone, which further worsen these pathophysiologic mechanisms, resulting in reduced serum sodium concentration. ${ }^{10}$ Following removal of excess peritoneal fluid, blood flow to the kidneys is initially improved, but ascitic fluid reaccumulates and the patient becomes intravascularly depleted. ${ }^{10}$

Infection is an important clinical mediator for the development of both portal hypertension as well as hyponatremia. Bacterial translocation leads to endotoxemia and increased tumor necrosis factor (TNF)alpha, resulting in increased splanchnic $\mathrm{NO}$ and splanchnic arterial vasodilatation. This process reduces cardiac output, which leads to increased AVP secretion. ${ }^{11,12}$ Endotoxin-mediated splanchnic vasodilatation, especially with spontaneous bacterial peritonitis (SBP), can adversely affect central blood volume status, especially in the presence of severe ascites. ${ }^{1}$ Clinicians providing care for patients with cirrhosis should be aware of these factors and closely monitor at-risk patients for the onset or worsening of hyponatremia. ${ }^{1}$

\section{PROGNOSTIC SIGNIFICANCE OF HYPONATREMIA IN CIRRHOSIS}

Hyponatremia has several important clinical implications for patients with cirrhosis. ${ }^{13}$ Hyponatremia is associated with refractory ascites, greater fluid accumulation, the need for paracentesis, and, importantly, impaired renal function. In patients with ascites and cirrhosis, approximately $50 \%$ have some degree of hyponatremia. ${ }^{2}$ Moreover, the severity of hyponatremia associated with advanced cirrhosis correlates with the degree of cirrhosis complications, especially hyponatremia associated with hepatorenal syndrome, encephalopathy, and SBP (Table 1). ${ }^{2}$

Similarly, hyponatremia is strongly associated with increasing Child-Pugh and Model for End-Stage Liver Disease (MELD) scores. ${ }^{14}$ In an analysis of data among candidates for liver transplantation from the Organ Procurement and Transplantation Network, the combination of MELD score and serum sodium concentration was a better predictor of death than the MELD score alone. ${ }^{14}$ In addition, the effect of hyponatremia on clinical outcomes was greater in patients
TABLE 1. Odds Ratio for Risk of Cirrhosis

Complications by Serum $\left[\mathrm{Na}^{+}\right]^{2}$

\begin{tabular}{llcll}
\hline & \multicolumn{3}{c}{ Serum [Na+] mEq/L } & $>135$ \\
\cline { 2 - 4 } & & $\leq 130$ & $131-135$ & \\
\hline Hepatorenal syndrome & 3.45 & 1.75 & 1 (reference value) & \\
Hepatic encephalopathy & 3.40 & 1.69 & 1 (reference value) \\
Gastrointestinal bleeding & 1.48 & 0.93 & 1 (reference value) & \\
Spontaneous bacterial peritonitis & 2.36 & 1.44 & 1 (reference value)
\end{tabular}

NOTE: With permission from John Wiley \& Sons. Angeli P et al. Hyponatremia in cirrhosis: Results of a patient population study. Hepatology. 2006;44:1535-1542. 02006 by the American Association for the Study of Liver Diseases.

with a low MELD score than those with a relatively high MELD score.. These results suggest that combining serum sodium concentrations with MELD scores to assign transplantation priority might reduce mortality among patients on the waiting list. ${ }^{14}$

Hyponatremia is also a marker for the development of overt hepatic encephalopathy in patients with cirrhosis. $^{13}$ One of the proposed mechanisms for encephalopathy is low-grade cerebral edema. This leads to the conversion of glutamate to glutamine by ammonia, which accumulates within astrocytes, causing astrocyte swelling and dysfunction. Because hyponatremia complicates the management of fluid overload, it increases the risk of developing or exacerbating hepatic encephalopathy. ${ }^{13}$

Hyponatremia is intimately involved with the development of renal failure in the patient with cirrhosis. It is an earlier and more sensitive marker of renal impairment and/or circulatory dysfunction than serum creatinine. ${ }^{15}$ It is often the precursor to the development of hepatorenal syndrome. ${ }^{16,17}$

Hyponatremia is more common in hospitalized versus ambulatory patients with cirrhosis. ${ }^{1}$ In a study of 126 patients with cirrhosis admitted to an intensive care unit, patients with serum $\left[\mathrm{Na}^{+}\right] \leq 135 \mathrm{mEq} / \mathrm{L}$ had a greater frequency of ascites, illness severity scores, hepatic encephalopathy, sepsis, renal failure, and inhospital mortality than normonatremic patients $(73.1 \%$ vs $55.9 \%) .{ }^{18}$ Persistent ascites and low serum sodium identified cirrhotic patients with a high mortality risk, despite low MELD scores, in a study of 507 veterans in the United States with cirrhosis. ${ }^{19}$ In a retrospective review of 127 patients, hyponatremia was predictive of the development of acute renal failure during hospitalization; among patients with hyponatremia who developed renal failure in the hospital, $72 \%$ died. $^{20}$

Clinical assessment of a patient with cirrhosis who has hyponatremia can be difficult. ${ }^{1}$ These patients have too much salt and water in the wrong spaces (ie, in the peritoneal cavity and peripheral tissue). As a result, it is possible to have fluid overload with intravascular depletion. A further complication is that dilutional hyponatremia is associated with hepatorenal syndrome. Because these patients have elevated blood urea nitrogen (BUN) and creatinine, and decreased 
urine output and urine sodium concentration, they appear to be indistinguishable from a patient with prerenal azotemia prior to volume expansion. ${ }^{1}$ Many of these factors and concerns are illustrated in the following case we handled several years ago.

\section{A 70-YEAR-OLD WOMAN WITH CIRRHOSIS}

K.R. is a 70-year-old white woman recently discharged from the hospital following treatment of recurrent cellulitis. Her past medical history is positive for cirrhosis secondary to active alcohol use, chronic autoimmune hepatitis, and iron overload. Her hospital course was notable for tense ascites, asterixis, and a serum $\left[\mathrm{Na}^{+}\right]$of $126 \mathrm{mEq} / \mathrm{L}$ at admission. K.R. was managed with large volume paracentesis with $25 \%$ salt-poor albumin, elevation of her lower extremities, discontinuation of diuretics, and $1 \mathrm{~L}$ fluid restriction. Her serum $\left[\mathrm{Na}^{+}\right]$increased to $128 \mathrm{mEq} / \mathrm{L}$. Although her cellulitis and edema both improved, both persisted. In addition, her mental status also improved, but asterixis persisted. At this point in the hospitalization, effective management of the cellulitis was hindered by the persistent edema, and its treatment with diuretics was limited by the hyponatremia and hepatic encephalopathy.

Today, we have better treatment options for managing this patient. To effectively correct the hyponatremia and facilitate treatment of the other complications of cirrhosis, we can now initiate therapy with one of the vaptans currently available.

\section{TREATMENT OF MILD ASYMPTOMATIC HYPERVOLEMIC HYPONATREMIA}

The initial approach to treatment of patients with mild asymptomatic, hypervolemic hyponatremia consists of fluid restriction and a sodium-restricted diet. ${ }^{1}$ Fluid restriction, however, has limited efficacy and is often not well tolerated by patients. For patients with severe or progressive hyponatremia, diuretics should be minimized or discontinued to avoid intravascular volume depletion. If patients have severe dilutional hyponatremia and tense ascites, therapeutic paracentesis with plasma expanders is safe. ${ }^{1}$

The pharmacologic approach to treating hyponatremia has advanced with the discovery of vaptans, drugs that inhibit $\mathrm{V}_{2}$ receptors in cells of the collecting ducts. ${ }^{21}$ In contrast to conventional diuretics, vaptans do not increase natriuresis. Administration of a vaptan agent for 1 to 2 weeks has been shown to significantly improve low serum sodium levels in patients with hyponatremia, and promote aquaresis without significantly altering renal or circulatory function or activity of the renin-angiotensin-aldosterone system. The most frequent side effect of vaptan therapy is thirst. ${ }^{21}$

Two vaptan agents are currently approved for use in the United States: conivaptan and tolvaptan. Conivap$\tan$ is administered intravenously, and is a nonselective vasopressin inhibitor, blocking both $\mathrm{V}_{1 \mathrm{~A}}$ and $\mathrm{V}_{2}$ receptors. The course of therapy for conivaptan is 4 days. Tolvaptan, on the other hand, selectively blocks $\mathrm{V}_{2}$ receptors, and is a once-daily oral vaptan that can be given long-term. ${ }^{21}$

The efficacy of tolvaptan was evaluated in the Study of Ascending Levels of Tolvaptan in Hyponatremia 1 and 2 (SALT-1 and SALT-2). ${ }^{22}$ In these multicenter, prospective, randomized, placebo-controlled trials, patients with dilutional hyponatremia (serum $\left[\mathrm{Na}^{+}\right]$ $<135 \mathrm{mEq} / \mathrm{L})$ associated with cirrhosis $(22.4 \%$ in SALT-1, $30.5 \%$ in SALT-2), heart failure, or syndrome of inappropriate antidiuretic hormone (ADH) hypersecretion, and who were hospitalized and clinically stable, received tolvaptan $15 \mathrm{mg}$ daily or placebo. Repeat serum sodium levels were obtained at 8 hours, 2,3 , and 4 days, and then weekly at days 11 , 18,25 , and 30 . The study drug was discontinued on day 30, with follow-up serum sodium levels taken 7 days later. (In patients with persistent hyponatremia, the tolvaptan dose was adjusted to $30 \mathrm{mg}$ and then 60 $\mathrm{mg}$ with the goal of achieving a serum $\left[\mathrm{Na}^{+}\right]<135$ $\mathrm{mEq} / \mathrm{L}$.) Increases in serum sodium concentration were seen as early as 8 hours after the first administration of tolvaptan and persisted throughout the study period. After tolvaptan was discontinued, serum sodium levels decreased to baseline within 1 week. ${ }^{22}$ Tolvaptan was well tolerated, with the most common side effects being increased thirst, dry mouth, and increased urination. ${ }^{22}$

Longer-term administration of tolvaptan was shown to maintain a higher serum sodium concentration with an acceptable safety profile in SALTWATER, the open-label extension of the SALT-1 and SALT-2 trials. ${ }^{23}$ The study included 111 patients with hyponatremia who received oral tolvaptan for a mean follow-up of 701 days. The most common adverse effects potentially related to tolvaptan were thirst, dry mouth, polydipsia, and polyuria. ${ }^{22,23}$ Overall, there were 9 possible and 1 probable serious adverse events, which represents an acceptable safety profile over 77,369 patient-days of exposure. Over time, 64 patients discontinued tolvaptan, 30 due to adverse reactions or death. $^{22}$ The results of SALTWATER indicated that most patients received benefit from treatment with tolvaptan, with a decreased need for fluid restriction. ${ }^{23}$

\section{PATIENT CHARACTERISTICS FOR TOLVAPTAN}

In the SALT trials, tolvaptan was administered to clinically stable patients. Based on recommendations by the US Food and Drug Administration (FDA), tolvaptan should be initiated or reinitiated in a hospital setting. ${ }^{1}$ Patients with severe neurologic symptoms due to hyponatremia should be treated with normal saline instead of tolvaptan; combination therapy with tolvaptan and normal saline should be avoided due to the potential for a too-rapid correction of hyponatremia and the potential for central pontine myelinolysis. 
Saline should be discontinued and persistent hyponatremia confirmed before beginning tolvaptan therapy. ${ }^{1}$

Several additional factors should be considered before patients begin tolvaptan. First, tolvaptan increases thirst, as well as the frequency and volume of urination. Therefore, patients must be able to respond appropriately to thirst with increased water intake. Patients should not be fluid-restricted during the first day of tolvaptan therapy; instead, they should be instructed to respond to their thirst with increased water ingestion. Because of these factors, caution should be exercised in administering tolvaptan to a confused, restrained patient. In addition, patients should have adequate toileting aids, such as a bedside urinal or commode. ${ }^{1}$

As with most new drugs, acquisition costs for tolvaptan should be considered in light of the clinical benefits of treatment outcomes. In a retrospective review, median hospital costs for patients with moderate-to-severe $(\$ 16,606)$ and mild-to-moderate hyponatremia $(\$ 14,266)$ were higher than matched patients without hyponatremia $(\$ 13,066) .{ }^{24}$ In the Efficacy of Vasopressin Antagonism in Heart Failure Outcome Study With Tolvaptan (EVEREST) trial, in which patients with severe congestive heart failure (including those with and without hyponatremia) were randomized to tolvaptan or placebo, the adjusted mean length of hospital stay for those with hyponatremia at baseline who received tolvaptan was 1.72 days shorter than those who received placebo. ${ }^{25}$ Although tolvaptan is somewhat expensive, the cost compares favorably with the daily cost of hospitalization.

\section{SUMMARY}

Portal hypertension plays a pivotal role in the development of hyponatremia in patients with cirrhosis. Reflex vasodilation in the splanchnic circulation compromises the effective central blood volume, triggering compensatory vasoconstrictor and antinatriuretic mechanisms. The net effect is greater free water accumulation than sodium retention, creating dilutional hyponatremia.

The severity of hyponatremia correlates with the severity of cirrhosis complications, such as hepatorenal syndrome, encephalopathy, SBP, and renal failure. The presence of hyponatremia is a marker for poor outcomes and shortened survival, regardless of MELD scores.

In a hospitalized, acutely ill patient with cirrhosis, such as the person in this case, therapy may involve discontinuation of diuretics, evaluation and treatment of infection, volume expansion with salt-poor albumin, and tolvaptan for treatment of hyponatremia. Regarding tolvaptan, early morning administration is recommended. At initiation of therapy, fluid restriction should be discontinued, and off-floor testing should be avoided. Concomitant medications should be reviewed to avoid potentially harmful interactions.

Disclosure: Advisory Board: Otsuka America Pharmaceutical, Inc.; Speaker's Bureau: Otsuka America Pharmaceutical, Inc. The author received support for travel and an honorarium from Paradigm Medical Communications for time and expertise spent writing this article.

\section{References}

1. Ross E, Sigal SH. Managing hyponatremia in cirrhosis. J Hosp Med. 2010;5(suppl 3):S8-S17.

2. Angeli $\mathrm{P}$, Wong $\mathrm{F}$, Watson $\mathrm{H}$, Ginès $\mathrm{P}$; for the CAPPS Investigators. Hyponatremia in cirrhosis: results of a patient population survey. $\mathrm{He}$ patology. 2006;44:1535-1542.

3. Shah V. Molecular mechanisms of increased intrahepatic resistance in portal hypertension. J Clin Gastroenterol. 2007;41(suppl 3): S259-S261.

4. Treiber G, Csepregi A, Malfertheiner P. The pathophysiology of portal hypertension. Dig Dis. 2005;23:6-10.

5. Iwakiri Y. The molecules: mechanisms of arterial vasodilatation observed in the splanchnic and systemic circulation in portal hypertension. J Clin Gastroenterol. 2007;41(suppl 3):S288-S294.

6. Iwakiri Y, Groszmann RJ. Vascular endothelial dysfunction in cirrhosis. J Hepatol. 2007;46:927-934.

7. Ginès $\mathrm{P}$, Cárdenas $\mathrm{A}$, Arroyo $\mathrm{V}$, et al. Management of cirrhosis and ascites. N Engl J Med. 2004;350(16):1646-1654.

8. Liamis G, Milionis H, Elisf M. A review of drug-induced hyponatremia. Am J Kidney Dis. 2008;52(1):144-153.

9. Solis-Herruzo JA, Moreno D, Gonzalez A, et al. Effect of intrathoracic pressure on plasma arginine vasopressin levels. Gastroenterology. 1991;101:607-617.

10. Ginès P, Tito L, Arroyo V, et al. Randomized comparative study of therapeutic paracentesis with and without intravenous albumin in cirrhosis. Gastroenterol. 1988;94:1493-1502.

11. Ros J, Clària J, To-Figueras J, et al. Endogenous cannabinoids: a new system involved in the homeostasis of arterial pressure in experimental cirrhosis in the rat. Gastroenterology. 2002;122:85-93.

12. Bátkai S, Mukhopadhyay P, Harvey-White J, et al. Endocannabinoids acting at CB1 receptors mediate the cardiac contractile dysfunction in in vivo in cirrhotic rats. Am J Physiol Heart Circ Physiol. 2007;293: H1689-H1695.

13. Häussinger D, Schliess F. Pathogenetic mechanisms of hepatic encephalopathy. Gut. 2008;57:1156-1165.

14. Kim WR, Biggins SW, Kremers WK, et al. Hyponatremia and mortality among patients on the liver-transplant waiting list. N Engl J Med. 2008;359:1018-1026.

15. Ruf AE, Kremers WK, Chavez LL, et al. Addition of serum sodium into the MELD score predicts waiting list mortality better than MELD alone. Liver Transpl. 2005;11:336-343.

16. Terg R, Gadano A, Cartier M, et al. Serum creatinine and bilirubin predict renal failure and mortality in patients with spontaneous bacterial peritonitis: a retrospective study. Liver Int. 2009;29:415-419.

17. Planas R, Montoliu S, Ballesté B, et al. Natural history of patients hospitalized for management of cirrhotic ascites. Clin Gastroenterol Hepatol. 2006;4:1385-1394.

18. Jenq CC, Tsai MH, Tian YC, et al. Serum sodium predicts prognosis in critically ill cirrhotic patients. J Clin Gastroenterol. 2010;44: $220-226$.

19. Heuman DM, Abou-Assi SG, Habib A, et al. Persistent ascites and low serum sodium identify patients with cirrhosis and low MELD scores who are at high risk for early death. Hepatology. 2004;40: 802-810.

20. Wu CC, Yeung LK, Tsai WS, et al. Incidence and factors predictive of acute renal failure in patients with advanced liver cirrhosis. Clin Nephrol. 2006;65:28-33.

21. Ginès $\mathrm{P}$, Guevara M. Hyponatremia in cirrhosis: pathogenesis, clinical significance, and management. Hepatology. 2008;48(3):1002-1010.

22. Schrier RW, Gheorghiade M, Berl T, et al. Tolvaptan, a selective oral vasopressin V2-receptor antagonist, for hyponatremia. N Engl J Med. 2006;355:2099-2112.

23. Berl T, Quittnat-Pelletier F, Verbalis JG, et al; for the SALTWATER Investigators. Oral tolvaptan is safe and effective in chronic hyponatremia. J Am Soc Nephrol. 2010;21:705-712.

24. Callahan MA, Do HT, Caplan DW, et al. Economic impact of hyponatremia in hospitalized patients: a retrospective cohort study. Postgrad Med. 2009;121(2):186-191.

25. Cyr PL, Slawsky KA, Olchanski N, et al. Effect of serum sodium concentration and tolvaptan treatment on length of hospitalization in patients with heart failure. Am J Health Syst Pharm. 2011;68(4): 328-333. 